\title{
Corona, Acute Ischemic Stroke, Malignant Cerebral Edema, and Hemo-adsorption: A Case Report
}

\author{
Mehul Shah ${ }^{1} \odot$, Zakaria Kaidawala ${ }^{\odot}$, Arun $_{\text {Shah }}^{3} \odot$, Rushi Desphande ${ }^{4} \odot$
}

\begin{abstract}
Background: COVID-19 infection can be associated with systemic hyperinflammation, hypercoagulable state, vasculitis, and cardiomyopathy leading to multiorgan failure. Use of extracorporeal blood purification has been shown to mitigate the cytokine storm, improving hemodynamic stability and pulmonary function.

Case summary: We report a case of a young patient with malignant cerebral edema due to acute cerebrovascular accident, with COVID-19. He was taken up for life-saving decompression craniotomy amidst the cytokine storm and multiorgan failure, and was treated with steroids, antibiotics, and Cytosorb ${ }^{\oplus}$ therapy for the cytokine storm. IL-6 and PCT levels were reduced by 99.5 and $98.6 \%$, respectively. Vasopressors were stopped on day 4 and successfully weaned off ventilator support by 2 weeks of tracheostomy. He was de-cannulated and discharged neurologically stable on day 32 .

Conclusion: Timely detection of COVID-19 and anti-inflammatory and hemo-adsorption measures may be helpful in modulating cytokine storm, thereby reducing morbidity and mortality.

Keywords: Acute ischemic stroke, Corona, COVID-19, Cytosorb, Hemoadsorption, Hemoperfusion, Malignant cerebral edema.

Indian Journal of Critical Care Medicine (2022): 10.5005/jp-journals-10071-24116
\end{abstract}

\section{INTRODUCTION}

Patients with COVID-19 have reported multiple manifestations including cerebrovascular accident and coagulopathies. ${ }^{1}$ In severe cases, a pronounced release of various cytokines has repeatedly been observed denoted as "cytokine storm," which can result in pronounced vasodilatation and membrane leakage, leading to severe, uncontrolled vasoplegic shock. ${ }^{2}$ Ruan et al. ${ }^{3}$ and Zhou et al. ${ }^{4}$ have identified high interleukin-6 (IL-6) levels as a potential predictor of a fatal outcome of COVID-19 disease. IL- 6 is also an important factor in the pathophysiology of severe septic shock and excessive immune response in hemophagocytic lymphohistiocytosis (HLH). ${ }^{5}$ In both these conditions, it is reported that the extracorporeal adsorption of IL- 6 and other inflammatory mediators via Cytosorb ${ }^{\circledR}$ adsorber (M/s CytoSorbents, Inc., New Jersey, USA) leads to a significant reduction of these cytokines in the patient's blood. ${ }^{6}$

Here, we report a case of a COVID-19 positive, young gentleman with massive stroke, malignant cerebral edema, and finally multisystem failure. He underwent early decompressive hemi-craniotomy and later on treatment with hemo-adsorption in the course of which he could not only successfully weaned off vasopressor support but finally also recovered from his multisystem failure and could be discharged from hospital after 32 days.

An informed consent from the patient and an approval from the institutional ethics committee (IEC) for the publication was taken. (Reference: IEC Study Number: HNH/IEC/2021/CR/CCM/06).

\section{Case Description}

A 29-year-old gentleman with diabetes mellitus has been presented to the emergency department with complaints of left sided upper limb and lower limb weakness associated with altered sensorium, vomiting, and hypotension. He was provisionally diagnosed with stroke, encephalopathy, and was suspected to be

\begin{abstract}
1,2 Department of Critical Care Medicine, Sir HN Reliance Foundation Hospital and Research Centre, Mumbai, Maharashtra, India

${ }^{3}$ Department of Neurosciences, Sir HN Reliance Foundation Hospital and Research Centre, Mumbai, Maharashtra, India

${ }^{4}$ Department of Nephro Critical Care, Sir HN Reliance Foundation Hospital and Research Centre, Mumbai, Maharashtra, India

Corresponding Author: Mehul Shah, Department of Critical Care Medicine, Sir HN Reliance Foundation Hospital and Research Centre, Mumbai, Maharashtra, India, Phone: +91 7710005285, e-mail: mehul.s.shah@rfhospital.org
\end{abstract}

How to cite this article: Shah M, Kaidawala Z, Shah A, Desphande R. Corona, Acute Ischemic Stroke, Malignant Cerebral Edema, and Hemoadsorption: A Case Report. Indian J Crit Care Med 2022;26(2):235-238.

Source of support: Nil

Conflict of interest: None

in septic shock. In the emergency department, he required high oxygen support to maintain saturation and was commenced on vasopressor support. Echocardiography was likely suggestive of Takotsubo or stress cardiomyopathy. He remained hypotensive with profound acidosis requiring escalation to two vasopressor support therapies. The noncontrast computed tomography brain revealed a massive right hemispheric infarct with raised intracranial pressure (ICP). He was immediately transferred to the intensive care unit, where he was resuscitated with fluid therapy, triple inotropes, broad spectrum antibiotics, and shock dose steroids. He developed anuria with persistent metabolic acidosis, for which he was commenced on continuous renal replacement therapy (RRT) along with the use of Cytosorb ${ }^{\circledR}$ adsorber (M/s CytoSorbents, Inc., New Jersey, USA). Over the next few hours his neurological status deteriorated from a GCS 14 to $7 / 15$ and was intubated for airway protection. A repeat CT brain (Fig. 1) revealed 
massively elevated ICP with midline shift due to malignant cerebral edema, requiring decompressive craniotomy.

He was diagnosed with COVID-19 positive with minimal respiratory involvement and was treated with only dexamethasone and continued on Cytosorb therapy along with dialysis. His condition deteriorated further to multiple organ failure. Inflammatory mediators (IL-6 5600 pg/mL, and PCT vs $198 \mathrm{ng} / \mathrm{dL}$ ) were pointing toward a cytokine storm, as seen in septic shock as well as critically ill COVID-19 patients; therefore, treatment with Cytosorb along with dialysis was continued. Subsequently over a period of 4 days IL-6 dropped to $99.5 \%$ (5600-23 pg/mL) and procalcitonin (PCT) to $98.6 \%(198-2.8 \mathrm{ng} / \mathrm{dL})$ and vasopressors were stopped. Figure 2 shows the serial drop of PCT over a week, correlating with drop of WBC counts. Serial fall in IL-6, D-dimer, and Ferritin is shown in Figure 3.
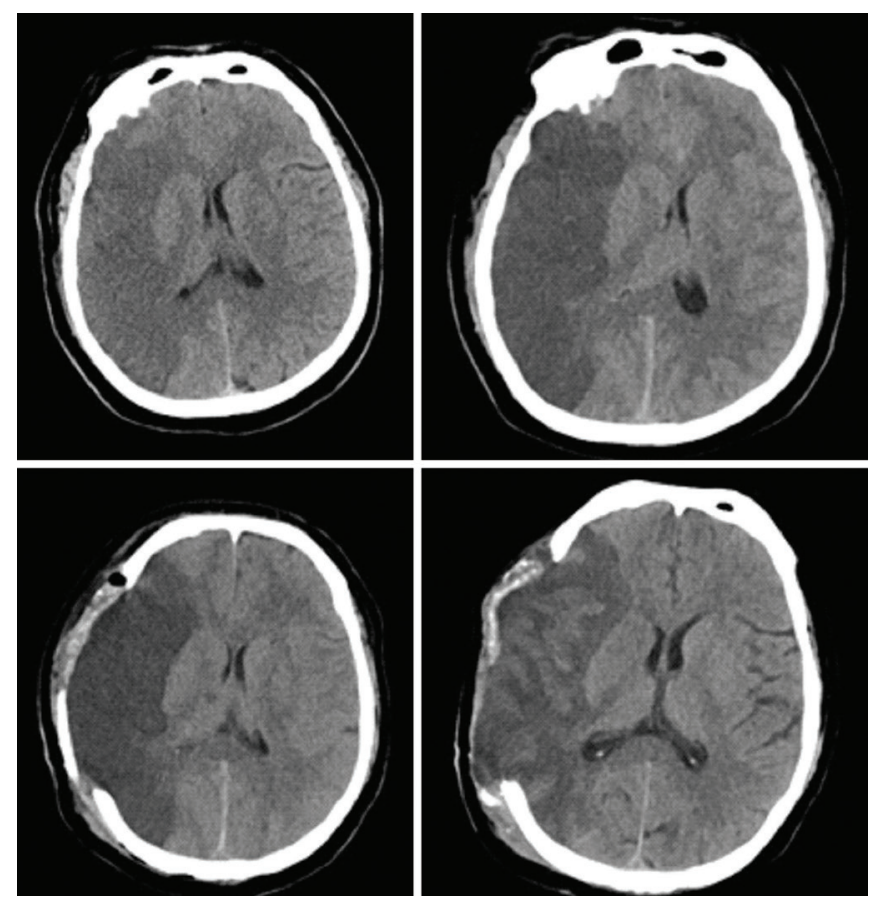

Fig. 1: CT images pre- and postcraniotomy

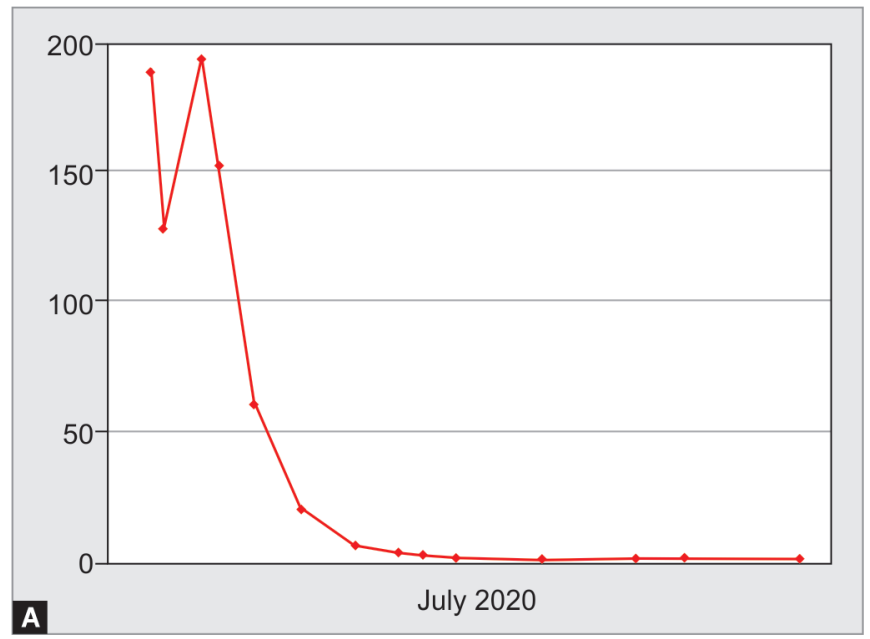

Figs $2 \mathrm{~A}$ and $\mathrm{B}$ : Change in procalcitonin and total leukocyte count (TLC)
The patient improved dramatically and his EF also normalized to $60 \%$. Regression in cerebral edema was seen on repeat CT brain and he started to obey commands but a left-sided hemiplegia persisted. All the bloody fluid cultures' and imaging for source of infection identification were negative.

Later, he developed purpura fulminans with digital necrosis and blistering of skin (Fig. 4); however, the Dopplers for lower limbs were normal. Unfortunately due to worsening gangrene probably owing to the hypercoagulable state of COVID-19 infection with diabetes he had to undergo both below knee amputations. A tracheostomy was performed in view of prolonged ventilation that weaned off ventilator support by 2 weeks. He also recovered from renal and liver dysfunctions by then. Neurologically he improved with good cognitive and bulbar functions but had leftsided hemiplegia. He was successfully de-cannulated and was discharged on day 32, on oral anticoagulants and anti-epileptics. On follow-up he is able to ambulate with prosthesis, awaiting cranioplasty.

\section{Discussion}

The innate response that commonly occurs in bacterial and viral infections is directed to combat and eliminate the infective pathogen. However, an uncontrolled, dysregulated immune response can also lead to injury to the host. Specifically in lungs, the profound inflammatory response may interfere with alveolar-capillary gas exchange, resulting in impaired oxygenation, an important cause of mortality in COVID-19. Heme-adsorption filters like Cytosorb/H-380 is a European Union (EU)-, FDA-, and DCGI-approved extracorporeal cytokine absorber, designed to broadly reduce "cytokine storm" and other inflammatory mediators in the blood that could otherwise lead to uncontrolled systemic inflammation, organ failure, and death in many life-threatening illnesses. ${ }^{8}$ In the present case, in addition to the steroids and antibiotics, Cytosorb therapy with dialysis may have played a significant role in reducing the cytokine storm.

Incidence of cerebrovascular incidents in COVID-19 is reported in the range of $1-6 \% .{ }^{9}$ Proposed mechanisms include prothrombotic state triggered by infliction of the endothelial cells by the virus itself ${ }^{10}$ and microangiopathic process due 


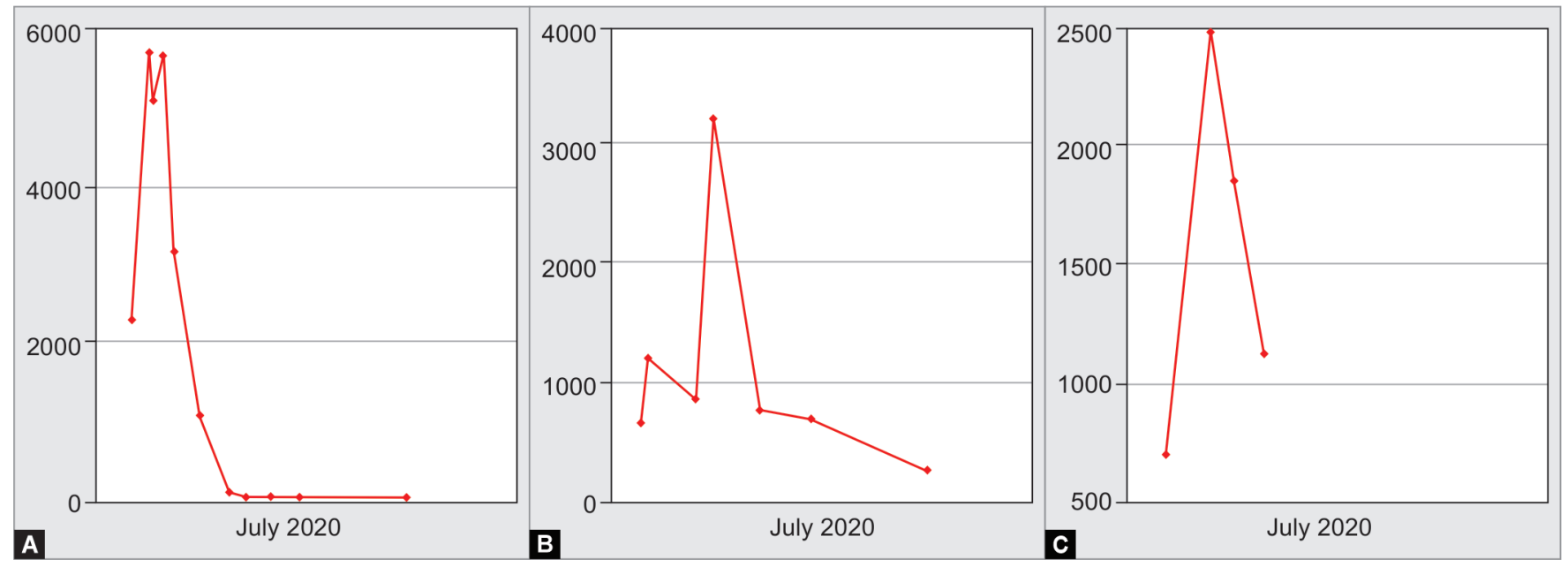

Figs $3 \mathrm{~A}$ to $\mathrm{C}$ : Change in IL-6, D-dimer, and ferritin
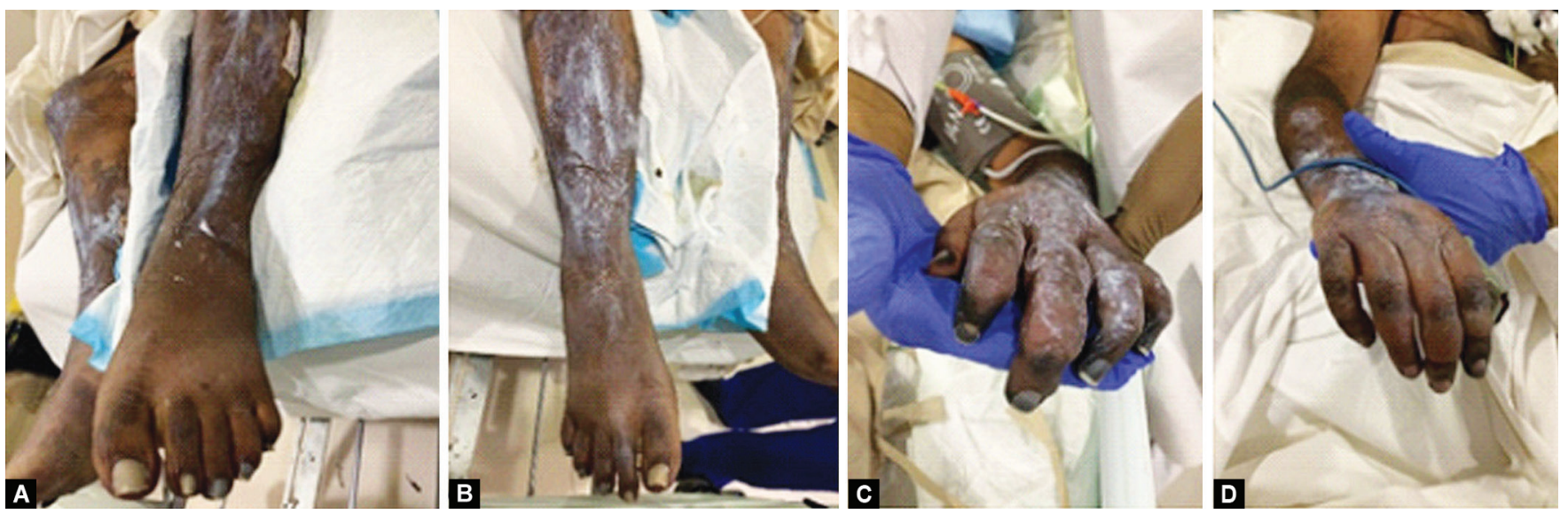

Figs 4A to D: Purpura fulminans with digital necrosis

to elevated C-reactive protein with $\mathrm{D}$-dimer. ${ }^{11}$ Large vessel occlusion (LVOs) are common in COVID-19 and more in younger patients. ${ }^{12}$ Decompressive craniotomy with good outcome has been reported in malignant cerebral edema in association with COVID-19 strokes by Liang et al. ${ }^{13}$ in three out of seven patients and by Sáez-Alegre et al. in a case of vertebral artery occlusion leading to cerebellar stroke. ${ }^{14}$

\section{CONCLUSION}

Our young patient with COVID-19, malignant cerebral edema amidst cytokine storm, and multiorgan failure was successfully treated with a standard AHA acute stroke management guideline for massive stroke and probably responded to anti-inflammatory and hemo-adsorption therapy given.

\section{ORCID}

Mehul Shah $\odot$ https://orcid.org/0000-0002-0720-7632

Zakaria Kaidawala @ https://orcid.org/0000-0003-4773-1030

Arun Shah 1 https://orcid.org/0000-0003-3383-7256

Rushi Desphande ํำ https://orcid.org/0000-0002-3969-1094

\section{References}

1. Spence JD, De Freitas GR, Pettigrew LC, Ay H, Liebeskind DS, Kase CS, et al. Mechanisms of stroke in COVID-19. Cerebrovasc Dis 2020;49(4):451-458. DOI: 10.1159/000509581.

2. Tang Y, Liu J, Zhang D, Xu Z, Ji J, Wen C. Cytokine storm in COVID-19: the current evidence and treatment strategies. Front Immunol 2020;11:1708. DOI: 10.3389/fimmu.2020.01708.

3. Ruan Q, Yang K, Wang W, Jiang L, Song J. Clinical predictors of mortality due to COVID-19 based on an analysis of data of 150 patients from Wuhan, China. Intensive Care Med 2020;46(5):846-848. DOI: 10.1007/s00134-020-05991-x.

4. Zhou F, Yu T, Du R, Fan G, Liu Y, Liu Z, et al. Clinical course and risk factors for mortality of adult inpatients with COVID-19 in Wuhan, China: a retrospective cohort study. Lancet 2020;395(10229): 1054-1062. DOI: 10.1016/S0140-6736(20)30566-3.

5. George MR. Hemophagocytic lymphohistiocytosis: review of etiologies and management. J Blood Med 2014;5:69-86. DOI: 10.2147/JBM.S46255.

6. Napp LC, Bauersachs J. Extracorporeal hemoperfusion: an option for COVID-19-associated cytokine storm syndrome. Shock 2020;54(5):700-701. DOI: 10.1097/SHK.0000000000001568.

7. Qin C, Zhou L, Hu Z, Zhang S, Yang S, Tao Y, et al. Dysregulation of Immune Response in Patients With Coronavirus 2019 (COVID-19) in Wuhan, China. Clin Infect Dis 2020;71(15):762-768. DOI: 10.1093/ cid/ciaa248. 
8. Rimmelé T, Kellum JA. Clinical review: blood purification for sepsis. Critical Care 2011;15(1):1-10. DOI: 10.1186/cc9411.

9. Ellul MA, Benjamin L, Singh B, Lant S, Michael BD, Easton A, et al. Neurological associations of COVID-19. Lancet Neurol 2020;19(9): 767-783. DOI: 10.1016/S1474-4422(20)30221-0.32.

10. Tahaghoghi-Hajghorbani S, Zafari P, Masoumi E, Rajabinejad M, Jafari-Shakib R, Hasani B, et al. The role of dysregulated immune responses in COVID-19 pathogenesis. Vir Res 2020;290:198197. DOI: 10.1016/j.virusres.2020.198197.

11. Merrill JT, Erkan D, Winakur J, James JA. Emerging evidence of a COVID-19 thrombotic syndrome has treatment implications. Nat Rev Rheumatol 2020;16:581-589. DOI: 10.1038/s41584-020-0474-5.
12. Oxley TJ, Mocco J, Majidi S, Kellner $\mathrm{CP}$, Shoirah $\mathrm{H}$, Singh IP, et al. Large-vessel stroke as a presenting feature of COVID-19 in the young. N Engl J Med 2020;382:e60. DOI: 10.1056/NEJMc2009787.

13. Liang JW, Reynolds AS, Kaitlin Reilly K, Lay C, Kellner CP. COVID-19 and decompressive hemicraniectomy for acute ischemic stroke. Stroke 2020;51:e215-e218. DOI: 10.1161/STROKEAHA.120. 030804.

14. Sáez-Alegre $M$, García-Feijoo P, Millán P, Vivancos Sánchez $C$, Rodríguez Domínguez V, García Nerín J, et al. Case report: decompressive craniectomy for COVID-19 malignant cerebral artery infarction. Is surgery a good option? Front Neurol 2021;12: 632036. DOI: 10.3389/fneur.2021.632036. 